\title{
Congenital portal vein aplasia with portocaval shunting in two dogs
}

\author{
Taesung Hwang ${ }^{1}$, Jonghyun Moon ${ }^{2}$, Hee Chun Lee ${ }^{1, *}$ \\ ${ }^{1}$ Department of Veterinary Medical Imaging, College of Veterinary Medicine, Gyeongsang National University, \\ Jinju 52828, Korea \\ ${ }^{2}$ Centum Animal Medical Center, Busan 48059, Korea
}

\begin{abstract}
Two dogs presented with vomiting and head pressing. In both dogs, a large vessel was revealed in computed tomography (CT) angiography, which was found to leave the portal vein (PV) cranial to the splenomesenteric confluence and enter the pre-hepatic caudal vena cava cranial to the right renal vein. The flow of portal blood to the liver was not identified. Based on CT angiography, the dogs were suspected to have congenital PV aplasia with portocaval shunting. Diagnostic imaging of potential malformations for PV continuation should be conducted before attempting shunt closure.
\end{abstract}

Keywords: CT angiography, dogs, portal vein aplasia, portosystemic shunt

\section{*Corresponding author}

Hee Chun Lee

Department of Veterinary Medical

Imaging, College of Veterinary Medicine, Gyeongsang National University, 501

Jinju-daero, Jinju 52828, Korea

Tel: $+82-55-772-2356$

Fax: $+82-55-772-2330$

E-mail: lhc@gnu.ac.kr

ORCID:

Hee Chun Lee

https://orcid.org/0000-0001-5936-9118

Taesung Hwang

https://orcid.org/0000-0001-6730-6061

Conflict of Interest

The authors declare no conflicts of interest.

Received: March 27, 2019

Revised: August 6, 2019

Accepted: August 19, 2019
The portosystemic shunt (PSS) is identified common vascular malformation in dogs [1]. The portal vein (PV) is formed by the confluence of the gastroduodenal, gastrosplenic, and cranial mesenteric veins. The formed PV travels toward the porta hepatis. On reaching the hilus of the liver, PV divides into right and left branches, which subsequently arborize to supply the hepatic lobes [2]. PSS is anomalous vascular communications from the PV to the systemic veins, bypassing the hepatic sinusoids, resulting in vomiting, stunted growth, hepatic encephalopathy, diarrhea, and cystic calculi [3]. Young cats and dogs may have either extrahepatic or intrahepatic shunts resulting from the developmental or congenital abnormalities of the portal or liver circulation, microvascular dysplasia, or PV aplasia [4,5].

Congenital aplasia of PV occurs uncommonly in dogs with PSS [3,6,7]. The aplasia of PV with the portal insertion into the caudal vena cava (CVC) is a severe form of congenital PSS [8]. The portal trunk insertion into CVC is uncommon form of PSS, and the connection between the CVC and the PV, like most shunts, occurs through a tributary of the PV [8].

The surgical closure of the shunt may be considered to treatment of dogs with PSS [9]. However, the surgical attenuation can result in acute shock and severe portal hypertension, because of aplasia or hypoplasia of the portal venous circulation cranial to the PSS [10]. Therefore, it is important to identify the dogs with PSS for continuity of PV before attempting surgical closure and PV interruption should be checked during surgical attenuation of PSS. When surgery is not possible, dogs with the clinical signs are managed medically, and treatment is directed at controlling the clinical signs. The mainstay of medical management is to reduce the amount of protein in the diet. If there is hepatic encephalopathy, treatment is based on the clinical signs [11]. The prognosis of portal aplasia is poor-to-guarded [5]. This report describes extrahepatic PSS with portal aplasia using computed tomography (CT) angiography in 2 dogs.

A 7-month-old, intact male Spitz (case 1) was referred to the Gyeongsang National University Veterinary Medical Teaching Hospital (Korea) with a history of decreased exercise tolerance, lack of appetite, and vomiting. In case 1, hematology and serum biochemistry analyses revealed leukocytosis $(22.8 \times$ $\left.10^{9} / \mathrm{L}\right)$ and elevated fasting serum bile acid concentration $(65 \mu \mathrm{mol} / \mathrm{L}$, reference range 0 to $10 \mu \mathrm{mol} / \mathrm{L}$ ), alanine aminotransferase (ALT) (205 U/L, refer- 
ence range 11 to $101 \mathrm{U} / \mathrm{L}$ ) and alkaline phosphatase (ALP) (552 U/L, reference range 22 to $211 \mathrm{U} / \mathrm{L}$ ) levels. A 2-yearold, intact female Maltese (case 2) was presented to the Centum Animal Medical Center with a 6-day history of lack of appetite, behavioral changes, and head pressing. The hematology analysis revealed leukocytosis $\left(33.9 \times 10^{9} / \mathrm{L}\right)$. Biochemistry profiles indicated increased liver enzymes (ALP $1,519 \mathrm{U} / \mathrm{L}$, reference range 22 to $211 \mathrm{U} / \mathrm{L}$; ALT $525 \mathrm{U} / \mathrm{L}$, reference range 11 to $101 \mathrm{U} / \mathrm{L})$, and hyperammonemia $(436 \mu \mathrm{mol} /$ $\mathrm{L}$, reference range 0 to $99 \mu \mathrm{mol} / \mathrm{L}$ ).

Radiographs for both dogs showed microhepatia and bladder calculus. In both cases, abdominal ultrasonography revealed a microhepatia and echogenicity of the liver parenchyma was normal. The PV branches within the hepatic parenchyma could not be identified. The shunting vessel was identified to arise from main PV and terminate in the CVC. A survey CT scan was obtained from the diaphragm to the coxofemoral joint. The following parameters: $110 \mathrm{kVp}, 120 \mathrm{~mA}$, gantry rotation time of $1-\mathrm{sec}$, slice thickeness of $2 \mathrm{~mm}$, pitches of 1.3. Dual-phase CT angiography was performed using nonionic (iohexol, $900 \mathrm{mg} / \mathrm{I} / \mathrm{kg}$ ) contrast medium by automatic power injector. The injection rate was $1 \mathrm{~mL} / \mathrm{s}$. Portal-phase scan time was obtained 50 -sec after the start of contrast medium injection. Images were identified with CT images analysis program with 3D capabilities (Lucion, Infinitt Technology, Korea). In both dogs, the hepatic artery originated from the celiac artery and followed a lateral course to reach the porta hepatis. the cranial mesenteric vein join to form the $\mathrm{PV}$, and PV traveled left laterally to insert into the prehepatic CVC. The portal trunk was tortuous and dilated. The pre-hepatic CVC was dilated at the shunt junction. The gastroduodenal and splenic vein traveled to join the PV. Continuation of the PV between the portal trunk and the liver was not identified in portal-phase CT angiography (Figs. 1 and 2 ). No other vascular abnormalities were revealed. The diagnosis in 2 dogs was aplasia of the PV with the portal insertion into the CVC.

In case 1 , the client did not want further treatment due to economic constraints. In case 2 , supportive treatment was given and symptomatic therapy was delivered by administration of L-ornithine-L-aspartate $(0.5 \mathrm{~mL} / \mathrm{kg}, \mathrm{PO}$; sid, HepaMerz; Han-Wha Pharm., Korea), metronidazole $(10 \mathrm{mg} / \mathrm{kg}$, PO, bid), lactulose (1 mL/kg, PO, bid), antioxidants, and liver protectants and the dog was discharged on a low protein diet. At reexamination 20 days later, clinical signs had improved and blood examination revealed that the white blood cell (WBC) concentration had decreased (WBC $12.7 \times 109 / \mathrm{L}$ ). Biochemistry profiles also indicated decreases in the liver enzymes (ALP $210 \mathrm{U} / \mathrm{L}$, reference range 22 to $211 \mathrm{U} / \mathrm{L}$; ALT $85 \mathrm{U} / \mathrm{L}$, reference range 11 to $101 \mathrm{U} / \mathrm{L})$, and ammonia level $(27 \mu \mathrm{mol} / \mathrm{L}$, reference range 0 to $99 \mu \mathrm{mol} / \mathrm{L})$.

Congenital PV aplasia with portal trunk insertion into the pre-hepatic CVC gave rise to clinical disease in 2 dogs. PV aplasia rarely occurs in humans [12,13]. Of the 135 dogs with PSS, 10 dogs have been identified as PV aplasia [14].
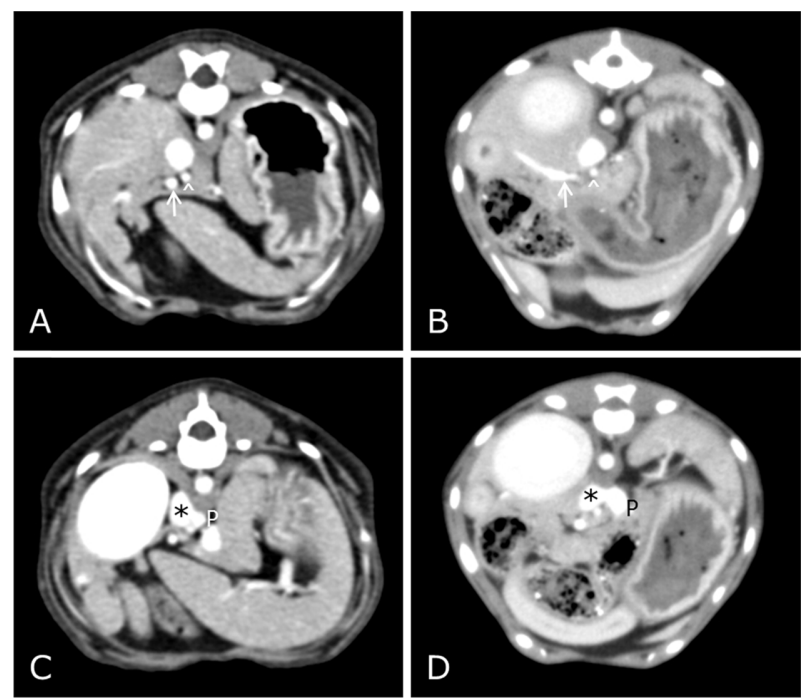

Fig. 1. Transverse computed tomography image of the abdomen of case 1 (A and C) and case 2 (B and D) during the portal phase. (A and B) The hepatic artery $\left({ }^{\wedge}\right)$ followed a lateral course to reach the porta hepatis. The gastroduodenal vein (arrows) is lateral to it. Either vessel could be mistaken for the portal vein. (C and D) The $\mathrm{P}$ inserts into the pre-hepatic caudal vena cava $(*)$ cranial to the right renal vein. $\mathrm{P}$, portal trunk.
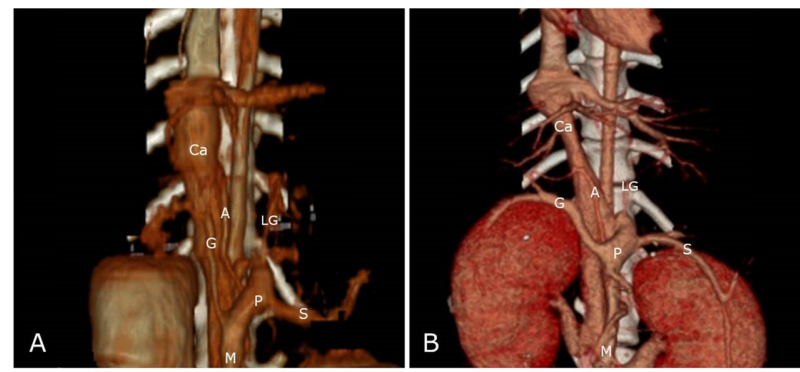

Fig. 2. Ventral view of the 3-dimensional rendering of the abdominal vasculature of case 1 (A) and case 2 (B) during the portal phase computed tomography scan. The cranial $M$ joins the enlarged $\mathrm{P}$. This inserts dorsally into the pre-hepatic $\mathrm{Ca}$. The $S$ and $G$ travel caudally to join the $P$, and no branches of the portal vein reach the liver.

A, hepatic artery; LG, left gastric artery; M, mesenteric vein; $\mathrm{Ca}$, caudal vena cava; S, splenic vein; $\mathrm{G}$, gastroduodenal vein; $\mathrm{P}$, portal trunk.

The portosystemic communication with PV aplasia cases were reported in relation to other vascular malformations [5]. In these reports, the PV interruption was accompanied by CVC continuity, suggesting an embryologic mechanism. During the developing embryo, there is continuation between the hepatic and the PVs, which ceases with the closure of the ductus venosus and development of the hepatic sinusoids [5]. Inappropriate anastomosis between the developing CVC and the vitelline veins, with portal trunk aplasia, results in porto- 
systemic anastomosis with PV interruption [5,8]. In other cases of PSS associated with interruption of PV, the PV entered the left hepatic vein due to failure of the ductus venosus to close and the persistence of the left vitelline vein [5,15]. Aplasia of PV with the portal trunk insertion into the CVC and the CVC interruption with communication as the azygos vein were also present $[5,8]$. The continuation of the CVC and azygos vein represents due to the atresia failure in the vessel segments connecting the 2 structures in the embryo development [8]. In our case, the portal trunk was interrupted cranially to the splenomesenteric confluence and inserted into the pre-hepatic CVC. No other vascular abnormalities were revealed.

One of the imaging challenges is to determine whether or not the PV travels to the liver. Either the gastroduodenal vein (GDV) or the enlarged hepatic artery can be mistaken for a hepatic PV due to difficulty tracing the vessels and mistiming of the scan phase [8]. Careful tracing of the GDV to the caudally located portal trunk, and the hepatic artery to the liver, is required for diagnosis [8]. Surgical attenuation and diagnostic imaging of PSS should be checked for other malformations because it is important to identify dogs with PSS for the PV continuation before surgical closure [5]. If aplasia of PV is present, the prognosis is poor-to-guarded [5]. In several reports, a vascular corrosion cast identified a hypoplastic vessel, which arise from the GDV and penetrate the liver. The hypoplastic vessel, which had not been identified at surgery, was suggested as a potential mechanism by which blood might reach the liver after surgical closure [5,15]. These reports suggest that the dogs with suspected aplasia of PV undergo portography during surgical closure to check the absence or presence of the portal blood flow to the liver. The diagnosis of aplasia of PV on CT should be performed with caution, since there may be a persistent small PV but it cannot be detected by CT and this vessel may dilate when blood flow is redirected surgically.

We described the imaging features of PV interruption with the PV insertion into the CVC in 2 dogs. CT angiography provided a means for making this distinction and detailed the course. The absence of portal blood flow should impart an unfavorable long-term prognosis in dogs with PSS. Diagnostic imaging of PSS should involve checking for potential malformation because it is important to evaluate continuation of PV before attempting shunt occlusion.

\section{References}

1. Buchanan JW. Causes and prevalence of cardiovascular disease. In: Kirk RW, Bonagura JD (eds.). Current Veterinary Therapy XI. pp. 647, PA Saunders, Philadelphia, 1992.

2. Evans HE. Veins. In: Evans HE (ed.). Miller's Anatomy of the Dog. 3rd ed. pp. 699, PA Saunders, Philadelphia, 1993.

3. Watson PJ, Herrtage ME. Medical management of congenital portosystemic shunts in 27 dogs--a retrospective study. J Small Anim Pract 1998;39:62-68.

4. Hunt GB. Effect of breed on anatomy of portosystemic shunts resulting from congenital diseases in dogs and cats: a review of 242 cases. Aust Vet J 2004;82:746-749.

5. Hunt GB, Bellenger CR, Borg R, Youmans KR, Tisdall PL, Malik R. Congenital interruption of the portal vein and caudal vena cava in dogs: six case reports and a review of the literature. Vet Surg 1998;27:203-215.

6. Breznock EM. Surgical manipulation of portosystemic shunts in dogs. J Am Vet Med Assoc 1979;174:819-826.

7. Hickman J, Edwards JE, Mann FC. Venous anomalies in a dog; absence of the portal vein; continuity of lower part of inferior vena cava with the azygos vein. Anat Rec 1949; 104:137-146.

8. Zwingenberger AL, Spriet M, Hunt GB. Imaging diagnosisportal vein aplasia and interruption of the caudal vena cava in three dogs. Vet Radiol Ultrasound 2011;52:444-447.

9. Hottinger HA, Walshaw R, Hauptman JG. Long-term results of complete and partial ligation of congenital portosystemic shunts in dogs. Vet Surg 1995;24:331-336.

10. Kummeling A, Van Sluijs FJ, Rothuizen J. Prognostic implications of the degree of shunt narrowing and of the portal vein diameter in dogs with congenital portosystemic shunts. Vet Surg 2004;33:17-24.

11. Berent AC. Hepatic vascular anomalies. In: Ettinger SJ, Feldman EC (eds.). Textbook of Veterinary Internal Medicine. 7th ed. pp. 1649-1672, Elsevier, St. Louis, 2010.

12. Komatsu S, Nagino M, Hayakawa N, Yamamoto H, Nimura Y. Congenital absence of portal venous system associated with a large inferior mesenteric-caval shunt: a case report. Hepatogastroenterology 1995;42:286-290.

13. Laverdiere JT, Laor T, Benacerraf B. Congenital absence of the portal vein: case report and MR demonstration. Pediatr Radiol 1995;25:52-53.

14. Center SA, Magne ML. Historical, physical examination, and clinicopathologic features of portosystemic vascular anomalies in the dog and cat. Semin Vet Med Surg (Small Anim) 1990; 5:83-93.

15. Lohse CL, Selcer RR, Suter PF. Hepatoencephalopathy associated with situs inversus of abdominal organs and vascular anomalies in a dog. J Am Vet Med Assoc 1976; 168:681-688 